\title{
Relationship between Fluorescein Pooling and Optical Coherence Tomographic Reflectivity of Cystoid Spaces in Diabetic Macular Edema.
}

\section{AUTHOR(S):}

Horii, Takahiro; Murakami, Tomoaki; Nishijima, Kazuaki; Akagi, Tadamichi; Uji, Akihito; Arakawa, Naoko; Muraoka, Yuki; Yoshimura, Nagahisa

\section{CITATION:}

Horii, Takahiro ...[et al]. Relationship between Fluorescein Pooling and Optical Coherence Tomographic Reflectivity of Cystoid Spaces in Diabetic Macular Edema.. Ophthalmology 2012, 119(5): 1047-1055

\section{ISSUE DATE:}

2012-05

URL:

http://hdl.handle.net/2433/155804

\section{RIGHT:}

(c) 2012 American Academy of Ophthalmology. Published by Elsevier Inc.; この論文は出版社版でありません。引用の際には出版社版をご確 認ご利用ください。; This is not the published version. Please cite only the published version. 
1 Relationship between Fluorescein Pooling and Optical Coherence Tomographic

2 Reflectivity of Cystoid Spaces in Diabetic Macular Edema

3

4 Takahiro Horii, MD, Tomoaki Murakami, MD, Kazuaki Nishijima, MD,

5 Tadamichi Akagi, MD, Akihito Uji, MD, Naoko Arakawa, MD, Yuki Muraoka, MD,

$6 \quad$ Nagahisa Yoshimura, MD

7

8 From the Department of Ophthalmology and Visual Sciences, Kyoto University Graduate

9 School of Medicine, Kyoto, Japan.

10

11 Meeting presentation: None

12 Financial support: None

13

14 Conflict of interest: No authors have any financial/conflicting interests to disclose.

16 Running head: Fluorescein Pooling and OCT Reflectivity in Diabetic CME

18 This article contains online-only material. The following should appear online-only: Table 2.

Correspondence to Tomoaki Murakami, MD, Department of Ophthalmology and Visual

Sciences, Kyoto University Graduate School of Medicine, 54 Shogoin-Kawaracho, Sakyo, Kyoto 606-8507, Japan; phone: 81-75-751-3250; fax: 81-75-752-0933; e-mail: mutomo@kuhp.kyoto-u.ac.jp. 
Horii et al.--2

\section{Abstract}

Objective: To study the characteristics of the reflectivity of the cystoid spaces and serous retinal detachment (SRD) on spectral-domain optical coherence tomography (SD-OCT) and the correlation with fluorescein findings in diabetic macular edema (DME). Design: Retrospective, observational, cross-sectional study.

Participants: Consecutive 134 eyes of 114 patients with clinically significant macular edema for whom SD-OCT and fluorescein angiography (FA) were performed on the same day.

Methods: FA using Heidelberg Retina Angiograph 2 and OCT images using Spectralis OCT were obtained. The reflectivity of the cystoid spaces and SRD on the OCT images was evaluated qualitatively and quantitatively and compared to the fluorescein pooling intensity on FA images.

Main Outcome Measures: The relationship between fluorescein pooling and the reflectivity characteristics of the cystoid spaces on SD-OCT images.

Results: One hundred forty-one cystoid spaces in 101 eyes were delineated on OCT images, and 138 (97.9\%) spaces had fluorescein pooling. Fifty-five (39.9\%) cystoid spaces with marked fluorescein pooling intensity had lower reflectivity on OCT images than those with modest pooling $(12.1 \pm 10.4$ vs. $22.0 \pm 15.4, P<0.001)$. The heterogeneity of the reflectivity of the cystoid spaces on the OCT images was associated significantly $(P<0.001)$ with modest fluorescein pooling. The hyperreflective foci in the cystoid spaces were correlated significantly with modest fluorescein pooling and higher or heterogeneous reflectivity on OCT images ( $P<0.001, P<0.001$, and $P=0.005$, respectively). In addition, the cystoid spaces with microaneurysms had higher or heterogeneous reflectivity on OCT images more frequently than those without microaneurysms $(P<0.001$ and $P=0.019$, 
Horii et al.--3

1 respectively). The reflectivity levels in the SRD were significantly $(P=0.005)$ lower than in

2 the cystoid spaces, and only one (3.3\%) eye had heterogeneous reflectivity on OCT images.

3 Conclusions: The results provided a novel interpretation of fluorescein pooling and

4 OCT characteristics of cystoid spaces and SRD in DME and suggested several mechanisms

5 by which the blood-retinal barrier is disrupted and concomitant edematous changes

6 develop.

7

8 
Horii et al.--4

\section{INTRODUCTION}

2 Diabetic macular edema (DME) and angiogenic complications are major causes of severe

3 visual loss in diabetic retinopathy (DR). ${ }^{1-2}$ Breakdown of the blood-retinal barrier (BRB) especially causes accumulation of intracellular or extracellular fluids and exacerbation of

5 neuroglial dysfunction and concomitant visual disruption. ${ }^{3-4}$ Although several therapeutic strategies have been reported, all are limited and many patients with DME still have poor

7 visual prognoses. ${ }^{5-10}$ This suggests several patterns of pathogenesis in DME, which remain to be elucidated.

The diagnosis of DME initially was based primarily on the results of slit-lamp biomicroscopy and fundus stereophotography. Another modality, fluorescein angiography (FA), delineates vascular lesions with higher contrast and various patterns of hyperfluorescence in the retinal parenchyma, i.e., focal or diffuse leakage and pooling with

13 petalloid or honeycomb-like patterns. ${ }^{11-12}$ The extravasation of fluorescein dye suggests a vascular pathophysiology and thickening of the retinal parenchyma. The major component of the inner BRB is believed to be retinal vascular endothelial cells with a highly integrated intercellular junctional complex, efflux transporters of wastes or toxic materials, and limited transcytosis, which are modulated by perivascular cells, pericytes, or glial cells. ${ }^{13-17}$ Diabetes exacerbates the breakdown of the BRB depending on several mechanisms: disrupted tight junctions, increased transcellular transports, and endothelial cell death, which are mediated via growth factors, cytokines, and several biochemical pathways. ${ }^{3-4,}$ ${ }^{18-20}$ Despite advances in basic research in vascular pathophysiology, the cellular or molecular mechanisms that determine the clinical fluorescein findings in DME remain unclear. 
1 images of the retinal pathology, has accelerated clinical research in DME. ${ }^{21-24}$ Time

2 domain-OCT delineates various morphologies in DME: serous retinal detachment (SRD),

3 cystoid macular edema (CME), and sponge-like retinal swelling. ${ }^{21}$ The objective

4 quantification using OCT identified a modest correlation between macular thickness and

$5 \quad$ visual acuity (VA) in DME. ${ }^{22}$ Recent technologic advances have resulted in spectral

6 domain (SD)-OCT, which improved the delineation of smaller structures with much higher

7 resolution. Fine lesions, i.e., hyperreflective foci, have been newly delineated on SD-OCT

8 images, and the detailed structural characteristics in microaneurysms have been

9 reported. ${ }^{25-27}$ Another advantage of SD-OCT with averaging processes is reduced speckle noise, which guarantees more accurate definition of reflectivity levels of the individual retinal layers and lesions. SD-OCT has been used to evaluate the external limiting membrane in DME and segmentation of the individual retinal layers. ${ }^{28-29}$

We evaluated qualitatively and quantitatively the reflectivity levels of the cystoid spaces and SRD on SD-OCT images and their relationship to hyperfluorescence in the images. We found that the fluorescein pooling intensity was related to the OCT reflectivity and its heterogeneity in the cystoid spaces in DME.

\section{Methods}

\section{Patients}

We retrospectively reviewed 134 eyes of 114 patients (age range, 33-89 years; mean, $65.0 \pm$ 10.8 years; 13 eyes with mild nonproliferative diabetic retinopathy [NPDR], 73 moderate NPDR, and 48 severe NPDR) who visited the Department of Ophthalmology in Kyoto University Hospital from May 2009 to February 2011. The inclusion criteria were eyes with clinically significant macular edema and those for whom FA and OCT images of 
Horii et al.--6

1 sufficient quality were obtained. Eyes with PDR were excluded, because intravitreal

2 hyperfluorescence from neovascular tissue makes it difficult to evaluate the exact levels of

3 intraretinal dye pooling. Since postoperative inflammation might modulate vascular

4 permeability, eyes in which cataract surgery were performed within 6 months and

5 vitrectomized eyes were also omitted. All research and measurements adhered to the tenets

6 of the Declaration of Helsinki. The ethics committee of our institution approved the study

7 protocol. Each patient provided informed consent after receiving a detailed explanation of

8 the nature and possible consequences of the study procedures.

\section{$9 \quad$ Optical Coherence Tomography}

10 After measuring the best-corrected VA (BCVA) and performing fundus biomicroscopy,

11 retinal sectional images of the macula were acquired and evaluated using SD-OCT

12 (Spectralis OCT, Heidelberg Engineering, Heidelberg, Germany) with infrared images.

13 Cross-hair scans (30 degrees) centered on the presumed fovea were obtained, and the

14 vertical images were used for further investigation. We then evaluated the contents of each

15 cystoid space and SRD within $1 \mathrm{~mm}$ of the center of the presumed fovea. The averaging

16 processes in SD-OCT reduce speckle noise and improve delineation of the pathological

17 structures, which encouraged us to evaluate the levels of reflectivity in each space qualitatively and quantitatively. The reflectivity in some spaces has heterogeneity either with or without a clear border, and others showed homogeneous reflectivity. Qualitative characteristics were estimated by two independent graders, and a third higher-level grader reviewed the images at the disagreement. To quantify the reflectivity levels, the margin of each space was manually traced using the images with inverted grayscale. The average reflectivity in the area encircled was measured using image processing software (Photoshop, Adobe Systems, San Jose, CA). We used the reflectivity levels of the vitreous cavity and 
6 relative reflectivity (A.U.) $=\frac{\text { reflectivity }(\text { cystoid space })-\text { reflectivity (vitreous) }}{\text { reflectivity }(\text { NFL })-\text { reflectivity }(\text { vitreous })} \times 100$

nerve fiber layer (NFL) as the standard in each image. After measuring the averaged reflectivity in each area as shown in the cystoid spaces, we defined the level in the vitreous as 0 and in the NFL as 100. The reflectivity values of the cystoid spaces or SRDs were calculated as an arbitrary unit (AU) according to the formula: 7.

The values were well agreed between two independent graders (intraclass correlation coefficient $[\mathrm{ICC}]=0.992$ ), and the average was applied to further analysis.

For an objective confirmation, we quantified OCT reflectivity of cytoid spaces automatically. We modified the method for automated detection of the margin of each cystoid space which was described previously. ${ }^{30}$ To be brief, after gray-scale TIFF images were imported into ImageJ (NIH, Bethesda, MD), an ImageJ plugin, E-Snake, was applied for the edge detection of cystoid spaces. We targeted each cystoid space using eight control points inside itself. The exact contour was determined by the optimization algorithm of the snake, followed by the calculation of the signal intensity in the encircled area of each cystoid space.

After the exclusion of two cystoid spaces in which the contour cannot be automatically decided, the reflectivity levels by this protocol were significantly correlated to those determined manually (ICC $=0.992$ ).

\section{Fluorescein Angiography}

Early- and late-phase FA images (6-10 minutes after intravenous injection) were obtained using Heidelberg Retinal Angiography 2 (HRA2, Heidelberg Engineering) as reported previously. ${ }^{24}$ We evaluated the pattern of hyperfluorescence on a vertical line that dissected 
Horii et al.--8

the presumed foveal center (within $1 \mathrm{~mm}$ centered on the presumed fovea) as focal, diffuse, or pooled (petalloid or honeycomb-like) as reported previously. ${ }^{11-12}$ The fluorescein pooling intensity then was divided into two levels. Briefly, after tracing the margin of each area of pooled dye, the fluorescein intensity was averaged using image processing software (Photoshop). Marked pooling indicated a higher level of fluorescein intensity than that of nearby venules, and modest pooling indicated a lower or the same level. Microaneurysms in fluorescein pooling were defined as those in the cystoid spaces.

In eyes with a SRD, we evaluated the presence of subretinal fluorescein pooling, which was defined as hyperfluorescence with a clear margin in the area corresponding to the SRD on the OCT images. Briefly, we first determined the presence of SRD at the presumed fovea and traced the subretinal spaces to the periphery until the margin of the SRD was identified. We then determined whether subretinal pooling of fluorescein dye was present. The margin of the pooled dye in the subretinal spaces should be very clear and round compared to the various patterns of hyperfluorescence in the retinal parenchyma.

\section{Statistical Analysis}

The results are expressed as the mean \pm standard deviation. The Student's $t$-test was used to compare the quantitative data populations with normal distributions and equal variance.

The data were analyzed using the Mann-Whitney $U$-test for populations with non-normal distributions or unequal variance. Significant differences in the sampling distribution were determined by Fisher's exact test. $P<0.05$ was considered statistically significant.

\section{Results}

The disrupted BRB in DR leads to several types of structural changes and accumulation of extracellular fluids. ${ }^{21}$ SD-OCT showed foveal cystoid spaces in 101 eyes and SRD in 30 
Horii et al.--9

eyes (Fig 1). We further investigated the patterns of the extracellular fluids in FA and SD-OCT in each lesion in DME.

\section{Association between Fluorescein Pooling and Optical Coherence Tomographic}

\section{Reflectivity of the Cystoid Spaces}

We first evaluated the fluorescein and OCT findings in 101 eyes with foveal cystoid spaces and found that 138 of 141 cystoid spaces delineated on OCT images had pooling of fluorescein. However, three cystoid spaces were not hyperfluorescent, and two were accompanied with epiretinal membrane (ERM) (Fig 2). The discrepancy in these cases suggests the limitation of FA for evaluating the cystoid spaces and mechanisms other than vascular hyperpermeability in the pathogenesis of the cystoid spaces.

Recent studies have reported that the morphologic patterns of hyperfluorescence are associated with the locations of the cystoid spaces in DME, which prompted us to investigate the relationship between fluorescein intensity and the OCT characteristics of 138 foveal cystoid spaces with fluorescein pooling. ${ }^{11-12}$ The average reflectivity in the cystoid spaces with marked fluorescein pooling was significantly lower than that with modest pooling $(P<0.001)$ (Figs 3, 4; Table 1, 2, available at http://aaojournal.org). Interestingly, 35 cystoid spaces showed heterogeneity in the reflectivity levels (Fig 3), which was significantly associated with modest fluorescein pooling $(P<0.001)$ (Table 1$)$.

\section{Hyperreflective Foci in the Cystoid Spaces}

The accumulation of hyperreflective foci in the subretinal spaces indicates a poor prognosis in DME, ${ }^{26}$ which encouraged us to investigate the characteristics of the hyperreflective foci in the foveal cystoid spaces. Curiously, their presence was associated significantly with modest fluorescein pooling $(P<0.001$; Table 3$)$. It seems inconsistent with the general belief that severe disruption of the BRB increases proteins, lipids, or lipid-laden macrophages and 
concomitant deposition of hard exudates in the retinal parenchyma. The cystoid spaces with hyperreflective foci had significantly higher levels of OCT reflectivity than those without foci $(P<0.001$; Table 3$)$. There also was a significant association between the hyperreflective foci and heterogeneity of the reflectivity in the cystoid spaces $(P=0.005$;

Table 3). These data suggested that higher reflectivity might represent condensed proteins or lipids, resulting in increased hyperreflective foci.

\section{Reflectivity in the Cystoid Spaces with Microaneurysms}

Microaneurysms are accompanied by vascular hyperpermeability and concomitant edema in the retinal parenchyma in DR. The association between microaneurysms and foveal cystoid spaces was reported recently, suggesting the contribution of microaneurysms to the pathogenesis of the cystoid spaces. ${ }^{24}$ When we investigated the OCT reflectivity in the cystoid spaces with microaneurysms, we found that these spaces had higher and heterogeneous reflectivity on OCT images more frequently than those without microaneurysms $(P<0.001$ and $P=0.019$, respectively) (Table 4$)$. This suggested that the disrupted BRB in microaneurysms often allowed the proteins and lipids to move into the cystoid spaces, at least in part. However, cystoid spaces with microaneurysms were not associated with fluorescein pooling intensity $(P=0.348)$ (Table 4).

\section{No Subretinal Fluorescein Pooling in Serous Retinal Detachments}

We investigated the characteristics of the FA and OCT findings in 30 eyes with SRD. Compared to the intraretinal hyperfluorescence in the parafoveal area, ${ }^{24}$ we did not find fluorescein pooling in the subretinal spaces (Fig 5). A further evaluation of the OCT images showed that the reflectivity levels in the SRD were significantly lower than in the cystoid spaces $(12.3 \pm 15.2$ vs. $18.6 \pm 15.2, P=0.005)$. The heterogeneity in OCT reflectivity was delineated in only one eye with SRD (3.3\%) compared to that in the cystoid spaces. The 
Horii et al.--11

1

2

3

4

5

6

7

differences in fluorescence and reflectivity on the OCT images suggested that the pathologic mechanisms in SRD differ from those in the cystoid spaces.

\section{Vitreomacular traction}

Vitreomacular traction (VMT) has been reported as another mechanism exacerbating DME, ${ }^{9,31-33}$ which prompted us to evaluate its association with fluorescein intensity and OCT reflectivity in cystoid spaces. Spectralis OCT delineated VMT including epiretinal membrane (ERM) in $19(18.8 \%)$ of 101 eyes with foveal cystoid spaces, and either qualitative or quantitative parameters in the contents of cystoid spaces were not associated with VMT (Table 5). Intriguingly, 16 (53.5\%) of 30 eyes with SRD were accompanied with VMT, whereas OCT delineated VMT in only 30 (28.8\%) of 104 eyes without SRD $(P=0.017)$. It suggests that tractional forces by posterior hyaloid might have an influence on the development or maintenance of SRD, at least in part.

\section{Discussion}

The current study showed for the first time the different levels of OCT reflectivity in the cystoid spaces in DME, suggesting the diversity of the contents. Increased reflectivity on the OCT images might be similar to flare in the intraocular humor, which can be seen by light reflection. In other words, the reflectivity could suggest the presence of concentrated proteins and lipids in the cystoid spaces. Pathohistology showed that hyaline deposits are often present in the cystoid spaces due to retinal vascular diseases, ${ }^{34-35}$ and the deposits might also have higher reflectivity on OCT images.

Barthelmes et al demonstrated the different levels of OCT reflectivity in cystoid spaces in different diseases, and speculated that the levels might be related to the pathogenesis of intraretinal spaces; exudation or degeneration. ${ }^{36}$ The heterogeneity in OCT 
Horii et al.--12

reflectivity was often delineated in cystoid spaces associated with retinal vascular diseases, whereas neuroglial degeneration in X-linked retinoschisis or high myopia results in lower and homogeneous reflectivity in intraretinal spaces. ${ }^{37-38}$ Considering the significant association between fluorescein intensity and OCT reflectivity in this current study, the diversity of OCT reflectivity levels in DME should depend on the different mechanisms in BRB breakdown, at least in part.

The physiologic BRB depends on a highly integrated intercellular junction complex, decreased transcellular transport, and active efflux of smaller molecules in the vascular endothelial cells, which are supported by perivascular components including the basement membrane, pericytes, and glial cells. ${ }^{13-17}$ These systems might be disrupted during the different stages of DR. Several pathological or biologic studies have reported that endothelial cell death, induced by inflammatory stimulation and biochemical pathways, increase the flux through the BRB in diabetes. ${ }^{19,39-40}$ Tight junctions were disrupted in diabetic rats or because of treatment with cytokines or growth factors, which results in increased paracellular flux and concomitant vascular hyperpermeability, ${ }^{18,41}$ and transcellular transport also was stimulated by growth factors, mediated via pinocytosis and vesiculovacuolar organelle. ${ }^{20}$

Endothelial cell death might be compatible with the current data that the cystoid spaces with higher reflectivity have lower fluorescein pooling intensity. ${ }^{19}$ We speculated that this kind of break in the retinal vasculature may allow the blood constituents to diffuse freely from or to the cystoid spaces, resulting in almost the same oncotic and hydrostatic pressure in the cystoid spaces as that in nearby capillaries. In other words, there are no active forces in transport into the cystoid spaces that depend on pressure differences. The amount of fluorescein moving into the cystoid spaces therefore decreased, with resultant 
Horii et al.--13

lower dye pooling intensity.

Partial disruption of the BRB might explain the findings that the cystoid spaces with lower reflectivity have higher intensity fluorescein pooling. Transgenic mice deleting claudin-5, an endothelial tight junction protein, had vascular hyperpermeability in smaller but not larger molecules. ${ }^{42}$ Several cellular and molecular mechanisms, result in the breakdown of tight junctions, which might permit smaller but not larger molecules to migrate through the capillary walls into the cystoid spaces. ${ }^{18,41-45}$ In such situations, Starling's equation, which describes the balance between hydrostatic and oncotic pressures, might be applied. ${ }^{46}$ Albumin, a main serum protein, could not move into the cystoid spaces, resulting in a difference in oncotic pressure between the cystoid spaces and the nearby capillaries. To neutralize it, the hydrostatic pressure in the capillaries should be higher than that in the cystoid spaces, which promotes movement of water and smaller molecules including fluorescein (molecular weight, approximately 330) into the cystoid spaces and increases the fluorescein pooling intensity.

Another explanation for the negative correlation between fluorescein intensity and reflectivity on OCT might be blockage or scattering of fluorescent light by the concentrated proteins. Based on this hypothesis, the negative correlation should be mathematically accurate, although we found some cystoid spaces without this association, suggesting that this explanation is not reasonable.

Interestingly, 35 cystoid spaces had heterogeneity in the reflectivity levels with clear borders. We speculated that higher reflectivity might correspond to blood clots or hyaline deposits in pathohistologic findings, ${ }^{34-35}$ whereas the areas with lower reflectivity might be filled with fluid. Among several possible components of hyaline deposits, most might be extravasated serum proteins or fibrins. We showed that heterogeneous reflectivity was 
Horii et al.--14

1 positively associated with modest fluorescein pooling. One explanation might be the

2 limited volume of the fluid phase, and another might be that the cystoid spaces are accompanied by severe disruption of the BRB and concomitant movement of serum proteins and lipids, as discussed previously. This theory might be supported by the positive association between the heterogeneous reflectivity and focal fluorescein leakage from microaneurysms, in which the vascular walls are sometimes ruptured. ${ }^{27}$

Hyperreflective foci, which may be precursors of hard exudates, were associated significantly with heterogeneity or higher levels of reflectivity in the cystoid spaces on the OCT images. In other words, the foci were observed in the cystoid spaces with severe disruption of the BRB. Although the origin of the hyperreflective foci remains controversial, ${ }^{25-26}$ condensed proteins or lipids in the cystoid spaces might deposit as hyperreflective foci, resulting in emergence of hard exudates. Another possibility is that lipid-laden macrophages might take up the concentrated proteins or lipids in the cystoid spaces. A severe break in the vascular walls might allow macrophage migration into the cystoid spaces, regardless of whether the BRB breakdown is or is not induced by inflammatory cells. ${ }^{19,39-40}$ In addition, considering the different appearances of the hyaline-like deposits and the hyperreflective foci on the OCT images, the foci may be lipid-laden macrophages rather than protein or lipid deposition. Since the Early Treatment Diabetic Retinopathy Study showed that the foveal hard exudates predict poor visual prognosis, ${ }^{47-48}$ clinicians should evaluate the OCT reflectivity in the foveal cystoid spaces, which also might exacerbate the subfoveal hard exudates.

Recent publications have reported an association between microaneurysms and cystoid spaces in DME. ${ }^{24,27}$ Cystoid spaces with microaneurysms had higher and heterogeneous reflectivity, suggesting that the BRB in the microaneurysms often was 
Horii et al.--15

damaged severely and allowed serum proteins and lipids to move into the cystoid spaces. SD-OCT showed the structural diversity in the vascular walls of the microaneurysms, and the absence or rupture of the thick walls often was associated with the heterogeneous reflectivity in the nearby cystoid spaces. In addition, circinate hard exudates often were seen around the microaneurysms in DR. We then speculated that microaneurysms, other vascular lesions, or mixed mechanisms contribute to the pathogenesis of the cystoid spaces with fluorescein pooling. A longitudinal study after direct coagulation of the microaneurysms might clarify this.

We did not see fluorescein pooling in the subretinal fluids in eyes with SRD associated with DME, compared to the breakdown of outer BRB in central serous chorioretinopathy (CSC). Intriguingly, VMT was significantly associated with SRD in DME, which might explain this discrepancy. We further found that cystoid spaces had higher levels of reflectivity on OCT images than SRD. These data seem inconsistent with the negative correlation between fluorescein pooling and OCT reflectivity in the cystoid spaces. Additionally, VMT did not affect the qualitative characteristics in the contents of cystoid spaces, although their volume might be exacerbated. No studies have definitively reported the pathogenesis of SRD development or maintenance in DME, although the data in the current study suggested that the pathogenesis of SRD differs at least partly from that of the cystoid spaces. Recent studies showed that the continuity from the intraretinal cystoid spaces to subretinal fluids in SRD due to branch retinal vein occlusion ${ }^{49}$ and the pathogenesis might be combined, after time passed by.

In the current study, we showed for the first time a negative correlation between fluorescein pooling intensity and OCT reflectivity of the cystoid spaces in DME, suggesting various patterns of BRB disruption. Recent advances in medicine and biology 
Horii et al.--16

1 have enabled application of several therapeutic strategies, although any treatment has only

2 partial effects on DME. Identification of several patterns of disruption of the BRB would

3 lead to development of customized therapies for DME.

4

5 
Horii et al.--17

\section{References}

2 1. Klein R, Klein BE, Moss SE, Cruickshanks KJ. The Wisconsin

3 Epidemiologic Study of Diabetic Retinopathy. XV. The long-term

4 incidence of macular edema. Ophthalmology 1995;102:7-16.

5 2. Aiello LP, Avery RL, Arrigg PG, et al. Vascular

6 endothelial growth factor in ocular fluid of patients with

7 diabetic retinopathy and other retinal disorders. N Engl J Med

$8 \quad 1994 ; 331: 1480-7$.

9 3. Gardner TW, Antonetti DA, Barber AJ, et al. Diabetic

10 retinopathy: more than meets the eye. Surv Ophthalmol

$112002 ; 47$ (suppl):S253-62.

12 4. Antonetti DA, Barber AJ, Bronson SK, et al, JDRF Diabetic

13 Retinopathy Center Group. Diabetic retinopathy: seeing beyond

14 glucose-induced microvascular disease. Diabetes

$15 \quad 2006 ; 55: 2401-11$.

16 5. Mohamed Q, Gillies MC, Wong TY. Management of diabetic

17 retinopathy: a systematic review. JAMA 2007;298:902-16.

18 6. Early Treatment Diabetic Retinopathy Study Research

19 Group. Photocoagulation for diabetic macular edema: Early

20 Treatment Diabetic Retinopathy Study report number 1. Arch

21 Ophthalmol 1985;103:1796-806.

22 7. Haritoglou C, Kook D, Neubauer A, et al. Intravitreal

23 bevacizumab (Avastin) therapy for persistent diffuse diabetic

24 macular edema. Retina 2006;26:999-1005. 


\section{Horii et al.--18}

1 8. Jonas JB, Sofker A. Intraocular injection of crystalline

2 cortisone as adjunctive treatment of diabetic macular edema.

3 Am J Ophthalmol 2001;132:425-7.

49 . Lewis H, Abrams GW, Blumenkranz MS, Campo RV. Vitrectomy

5 for diabetic macular traction and edema associated with

6 posterior hyaloidal traction. Ophthalmology 1992;99:753-9.

7 10. Aiello LP. The potential role of $\mathrm{PKC}$ beta in diabetic

8 retinopathy and macular edema. Surv Ophthalmol

$9 \quad 2002 ; 47$ (suppl):S263-9.

10 11. Otani T, Kishi S. Correlation between optical coherence

11 tomography and fluorescein angiography findings in diabetic

12 macular edema. Ophthalmology 2007;114:104-7.

13 12. Bolz M, Ritter M, Schneider M, et al. A systematic

14 correlation of angiography and high-resolution optical

15 coherence tomography in diabetic macular edema. Ophthalmology

$162009 ; 116: 66-72$.

17 13. Shakib M, Cunha-Vaz JG. Studies on the permeability of

18 the blood-retinal barrier. IV. Junctional complexes of the

19 retinal vessels and their role in the permeability of the

20 blood-retinal barrier. Exp Eye Res 1966;5:229-34.

21 14. Schinkel AH, Smit JJ, van Tellingen O, et al. Disruption

22 of the mouse mdrla P-glycoprotein gene leads to a deficiency

23 in the blood-brain barrier and to increased sensitivity to drugs.

24 Cell 1994;77:491-502. 
1 15. Coomber BL, Stewart PA. Three-dimensional reconstruction

2 of vesicles in endothelium of blood-brain barrier versus highly

3 permeable microvessels. Anat Rec 1986;215:256-61.

4 16. Cunha-Vaz J. The blood-ocular barriers. Surv Ophthalmol

$5 \quad 1979 ; 23: 279-96$.

6 17. Bernstein MH, Hollenberg MJ. Fine structure of the

7 choriocappillaris and retinal capillaries. Invest Ophthalmol

$8 \quad 1965 ; 4: 1016-25$.

9 18. Antonetti DA, Barber AJ, Khin S, et al, Penn State Retina

10 Research Group. Vascular permeability in experimental diabetes

11 is associated with reduced endothelial occludin content:

12 vascular endothelial growth factor decreases occludin in

13 retinal endothelial cells. Diabetes 1998;47:1953-9.

14 19. Joussen AM, Poulaki V, Mitsiades N, et al. Suppression

15 of Fas-FasL-induced endothelial cell apoptosis prevents

16 diabetic blood-retinal barrier breakdown in a model of

17 streptozotocin-induced diabetes. FASEB J 2003;17:76-8.

18 20. Hofman P, Blaauwgeers HG, Tolentino MJ, et al. VEGF-A

19 induced hyperpermeability of blood-retinal barrier

20 endothelium in vivo is predominantly associated with

21 pinocytotic vesicular transport and not with formation of

22 fenestrations. Curr Eye Res 2000;21:637-45.

23 21. Otani T, Kishi S, Maruyama Y. Patterns of diabetic macular

24 edema with optical coherence tomography. Am J Ophthalmol 
$1 \quad 1999 ; 127: 688-93$.

2 22. Diabetic Retinopathy Clinical Research Network.

3 Relationship between optical coherence tomography-measured

4 central retinal thickness and visual acuity in diabetic macular

5 edema. Ophthalmology 2007;114:525-36.

6 23. Sakamoto A, Nishijima K, Kita M, et al. Association

7 between foveal photoreceptor status and visual acuity after

8 resolution of diabetic macular edema by pars plana vitrectomy.

9 Graefes Arch Clin Exp Ophthalmol 2009;247:1325-30.

10 24. Murakami T, Nishijima K, Sakamoto A, et al. Foveal cystoid spaces are associated with enlarged foveal avascular zone and microaneurysms in diabetic macular edema. Ophthalmology $2010 ; 118: 359-67$

14 25. Bolz M, Schmidt-Erfurth U, Deak G, et al, Diabetic

15 Retinopathy Research Group Vienna. Optical coherence

16 tomographic hyperreflective foci: a morphologic sign of lipid

17 extravasation in diabetic macular edema. Ophthalmology

$18 \quad 2009 ; 116: 914-20$.

19 26. Ota M, Nishijima K, Sakamoto A, et al. Optical coherence

20 tomographic evaluation of foveal hard exudates in patients with

21 diabetic maculopathy accompanying macular detachment.

22 Ophthalmology 2010;117:1996-2002.

23 27. Horii T, Murakami T, Nishijima K, et al. Optical coherence

24 tomographic characteristics of microaneurysms in diabetic 
Horii et al.--21

1 retinopathy. Am J Ophthalmol 2010;150:840-8.

2 28. Murakami T, Nishijima K, Sakamoto A, et al. Association

3 of pathomorphology, photoreceptor status, and retinal

4 thickness with visual acuity in diabetic retinopathy. Am J

$5 \quad$ Ophthalmol 2011;151:310-7.

6 29. Tan O, Chopra V, Lu AT, et al. Detection of macular

7 ganglion cell loss in glaucoma by Fourier-domain optical

8 coherence tomography. Ophthalmology 2009;116:2305-14.

9 30. Fernandez DC. Delineating fluid-filled region boundaries

10 in optical coherence tomography images of the retina. IEEE Trans

11 Med Imaging 2005;24:929-45.

12 31. Kaiser PK, Riemann CD, Sears JE, Lewis H. Macular traction

13 detachment and diabetic macular edema associated with posterior

14 hyaloidal traction. Am J Ophthalmol 2001;131:44-9.

15 32. Diabetic Retinopathy Clinical Research Network Writing

16 Committee. Vitrectomy outcomes in eyes with diabetic macular

17 edema and vitreomacular traction. Ophthalmology

$18 \quad 2010 ; 117: 1087-93$.

19 33. Bhagat N, Grigorian RA, Tutela A, Zarbin MA. Diabetic

20 macular edema: pathogenesis and treatment. Surv Ophthalmol

$21 \quad 2009 ; 54: 1-32$.

22 34. Tsomo. Pathology of cystoid macular edema. Ophthalmology

$231982 ; 89: 902-15$.

24 35. Wolter JR. The histopathology of cystoid macular edema. 
1 Albrecht Von Graefes Arch Klin Exp Ophthalmol 1981;216:85-101.

2 36. Barthelmes D, Sutter FK, Gillies MC. Differential optical

3 densities of intraretinal spaces. Invest Ophthalmol Vis Sci

$4 \quad 2008 ; 49: 3529-34$.

5 37. Gregori NZ, Berrocal AM, Gregori G, et al. Macular

6 spectral-domain optical coherence tomography in patients with

7 X linked retinoschisis. Br J Ophthalmol 2009;93:373-8.

8 38. Benhamou N, Massin P, Haouchine B, et al. Macular

9 retinoschisis in highly myopic eyes. Am J Ophthalmol

$10 \quad 2002 ; 133: 794-800$.

11 39. Kowluru RA. Effect of advanced glycation end products on accelerated apoptosis of retinal capillary cells under in vitro conditions. Life Sci 2005;76:1051-60.

14 40. Nishikawa T, Edelstein D, Du XL, et al. Normalizing

15 mitochondrial superoxide production blocks three pathways of

16 hyperglycaemic damage. Nature 2000;404:787-90.

17 41. Murakami T, Felinski EA, Antonetti DA. Occludin

18 phosphorylation and ubiquitination regulate tight junction

19 trafficking and vascular endothelial growth factor-induced

20 permeability. J Biol Chem 2009;284:21036-46.

21 42. Nitta $T$, Hata M, Gotoh $\mathrm{S}$, et al. Size-selective loosening

22 of the blood-brain barrier in claudin-5-deficient mice. J Cell

23 Biol 2003;161:653-60.

24 43. Hammes HP, Lin J, Wagner P, et al. Angiopoietin-2 causes 


\section{Horii et al.--23}

1 pericyte dropout in the normal retina: evidence for involvement

2 in diabetic retinopathy. Diabetes 2004;53:1104-10.

344. Armulik A, Genove G, Mae M, et al. Pericytes regulate the

4 blood-brain barrier. Nature 2010;468:557-61.

5 45. Furuse M, Tsukita S. Claudins in occluding junctions of

6 humans and flies. Trends Cell Biol 2006;16:181-8.

746. Salathe EP, Venkataraman R. Interaction of fluid movement

8 and particle diffusion across capillary walls. J Biomech Eng

$9 \quad 1982 ; 104: 57-62$.

10 47. Chew EY, Klein ML, Ferris FL III, et al, ETDRS Research

11 Group. Association of elevated serum lipid levels with retinal

12 hard exudate in diabetic retinopathy: Early Treatment Diabetic

13 Retinopathy Study (ETDRS) report 22. Arch Ophthalmol

$14 \quad 1996 ; 114: 1079-84$.

15 48. Fong DS, Segal PP, Myers F, et al, Early Treatment Diabetic

16 Retinopathy Study Research Group. Subretinal fibrosis in

17 diabetic macular edema: ETDRS report 23. Arch Ophthalmol

$18 \quad 1997 ; 115: 873-7$.

19 49. Tsujikawa A, Sakamoto A, Ota M, et al. Serous retinal

20 detachment associated with retinal vein occlusion. Am J

21 Ophthalmol 2010;149:291-301. 


\section{TABLES}

Table 1. Correlation between fluorescein pooling and optical coherence tomographic characteristics of cystoid spaces in diabetic macular edema

\begin{tabular}{l|cc|c} 
& $\begin{array}{c}\text { fluorescein pooling } \\
\text { marked }\end{array}$ & $\begin{array}{c}\text { fluorescein pooling } \\
\text { modest }\end{array}$ & $p$-value \\
\hline $\begin{array}{l}\text { reflectivity levels in cystoid } \\
\text { spaces on OCT images } \\
\text { reflectivity patterns in cystoid }\end{array}$ & $12.1 \pm 10.4$ & $22.0 \pm 15.4$ & $<0.001$ \\
spaces & & & \\
homogeneous & 52 & 51 & $<0.001$ \\
heterogeneous & 3 & 32 &
\end{tabular}

OCT $=$ optical coherence tomography. 
Table 3. Hyperreflective foci in cystoid spaces in diabetic macular edema

\begin{tabular}{|c|c|c|c|}
\hline & $\begin{array}{c}\text { hyperreflective foci in } \\
\text { cystoid spaces } \\
\text { present }\end{array}$ & $\begin{array}{c}\text { hyperreflective foci in } \\
\text { cystoid spaces } \\
\text { absent }\end{array}$ & $p$-value \\
\hline \multicolumn{4}{|l|}{ fluorescein pooling } \\
\hline marked & 13 & 41 & \\
\hline modest & 48 & 36 & $<0.001$ \\
\hline reflectivity levels in cystoid & $23.8 \pm 11.3$ & $13.6 \pm 15.2$ & $<0.001$ \\
\hline \multicolumn{4}{|l|}{ spaces on OCT images } \\
\hline \multicolumn{4}{|l|}{ reflectivity patterns in } \\
\hline & & & \\
\hline homogeneous & 38 & 65 & \\
\hline heterogeneous & 23 & 12 & 0.005 \\
\hline
\end{tabular}

OCT $=$ optical coherence tomography . 
Table 4. Association between microaneurysms and optical coherence tomographic reflectivity in diabetic macular edema

\begin{tabular}{l|cc|c} 
& $\begin{array}{c}\text { microaneurysms in } \\
\text { fluorescein pooling } \\
\text { present }\end{array}$ & $\begin{array}{c}\text { microaneurysms in } \\
\text { fluorescein pooling } \\
\text { absent }\end{array}$ & $p$-value \\
\hline $\begin{array}{c}\text { fluorescein pooling } \\
\text { marked }\end{array}$ & 14 & 40 & \\
$\begin{array}{l}\text { modest } \\
\text { reflectivity levels in cystoid }\end{array}$ & $23.4 \pm 12.8$ & 55 & 0.348 \\
spaces on OCT images & & $16.3 \pm 15.7$ & $<0.001$ \\
reflectivity patterns & & & \\
in cystoids spaces & 26 & & \\
$\quad$ homogeneous \\
heterogeneous
\end{tabular}

OCT $=$ optical coherence tomography 
Table 5. Fluorescein pooling or optical coherence tomographic characteristics in cystoid spaces with vitreomacular traction in diabetic macular edema

\begin{tabular}{|c|c|c|c|}
\hline & $\operatorname{VMT}(+)$ & VMT (-) & $p$-value \\
\hline fluorescein pooling & & & \\
\hline marked & 11 & 43 & \\
\hline modest & 11 & 73 & 0.341 \\
\hline $\begin{array}{l}\text { reflectivity levels in cystoid spaces } \\
\text { on OCT images } \\
\text { reflectivity patterns in cystoid spaces }\end{array}$ & $14.9 \pm 9.8$ & $18.7 \pm 15.2$ & 0.301 \\
\hline homogeneous & 17 & 86 & \\
\hline heterogeneous & 5 & 30 & 1.000 \\
\hline hyperreflecitve foci in cystoid spaces & & & \\
\hline present & 8 & 53 & \\
\hline absent & 14 & 63 & 0.488 \\
\hline microaneurysms in fluorescein poolin & & & \\
\hline present & 5 & 38 & \\
\hline absent & 17 & 78 & 0.455 \\
\hline
\end{tabular}

$\mathrm{VMT}=$ vitreomacular traction.

OCT $=$ optical coherence tomography. 


\section{FIGURE LEGENDS}

Figure 1. Three patterns of foveal pathomorphologies in diabetic macular edema. Spectralis optical coherence tomography delineated serous retinal detachment (A), cystoid macular edema (B), and retinal swelling (C) at the presumed foveal center. Arrow: presumed foveal center. Scale bar $=200 \mu \mathrm{m}$.

Figure 2. Fluorescein pooling and cystoid spaces delineated by optical coherence tomography.

Fluorescein angiography in the late phase (B) showed fluorescein pooling at the fovea, compared to the early phase (A). (C) A cystoid space was delineated on the optical coherence tomography (OCT) image, in the area corresponding to the pooling. (F) In several eyes, cystoid spaces on OCT image were not accompanied with hyperfluorescence in both the early (D) and late (E) phases. OCT images were dissecting along the white arrows in the late phases. Black arrow: cystoid spaces.

Figure 3. Various patterns of optical coherence tomographic reflectivity in cystoid spaces.

(A) A cystoid space had the homogeneous and lower reflectivity on optical coherence tomography image. (C) A cystoid space with homogeneous and higher reflectivity was accompanied with hyperreflective foci. (E) A cystoid space showed the heterogeneity in reflectivity. Cystoid spases were traced and encircled (black curved line), followed by the quantification of the average reflectivity, as shown in the Methods section. The relative reflectivity in each cystoid space was -1.3 (B), 108.9 (D), or 23.8 (F). Arrows: cystoid spaces. Arrowheads: hyperreflective foci. Scale bar $=200 \mu \mathrm{m}$.

Figure 4. Negative correlation between fluorescein intensity in pooling and optical coherence tomographic reflectivity in cystoid spaces.

Fluorescein angiography in the late phase (B) showed marked intensity of fluorescein pooling (arrowheads), compared to the early phase (A), and corresponding cystoid spaces (arrowheads) had lower reflectivity on optical coherence tomography (OCT) image (E). (D) Fluorescein intensity in pooling was modest in the late phase (arrowheads), whereas dye pooling was absent in the early phase (C). The corresponding cystoid space (arrowheads) showed higher reflectivity with hyperreflective foci (black arrows) on OCT image (F). OCT images were dissecting along the white arrows in the late phases.

Figure 5. No fluorescein pooling in serous retinal detachment.

Fluorescein angiography in the early (A) or late phase (B) did not show fluorescein pooling in the area corresponding to serous retinal detachment delineated on optical coherence tomography image (C). 


\section{A}

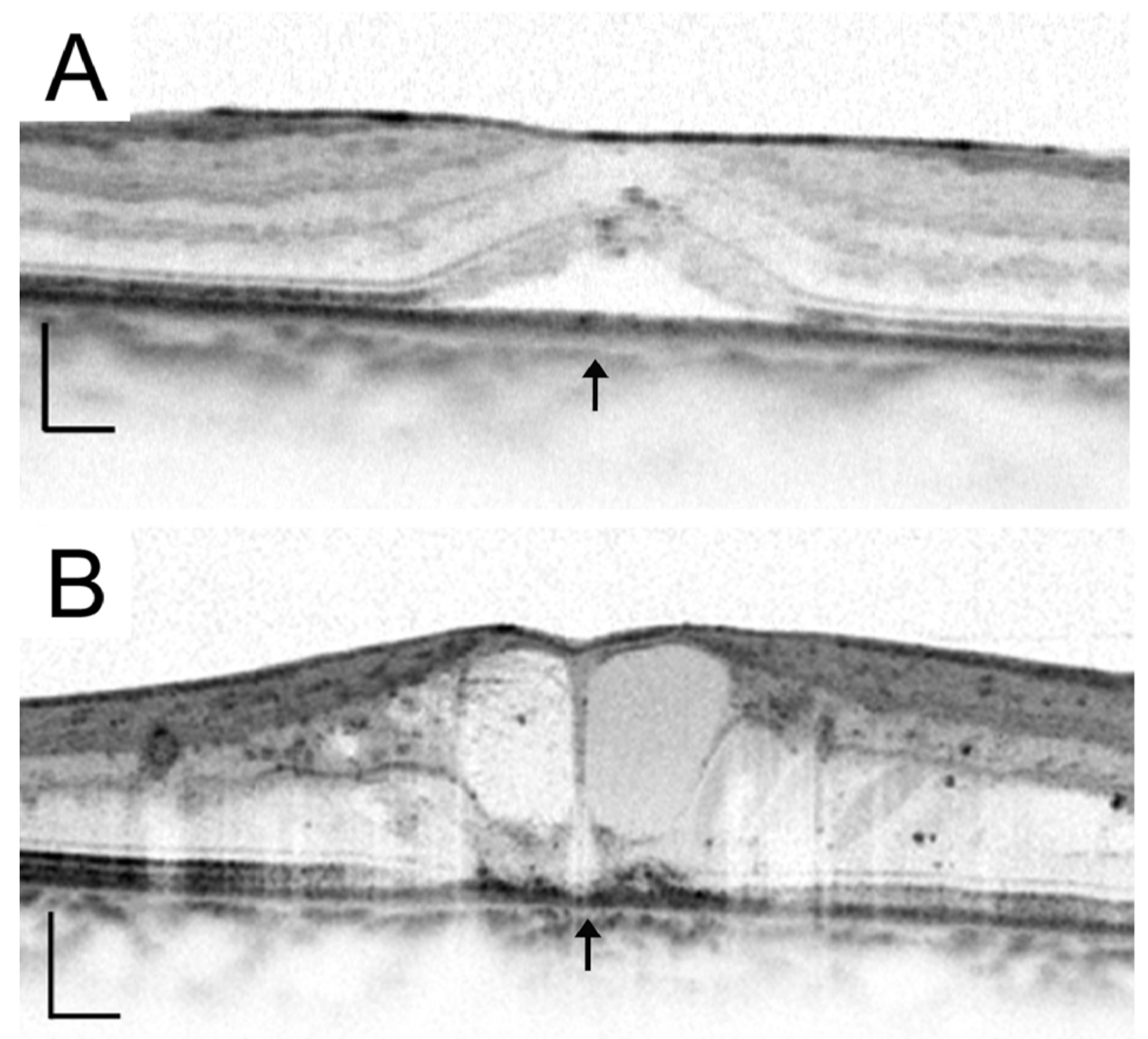

B
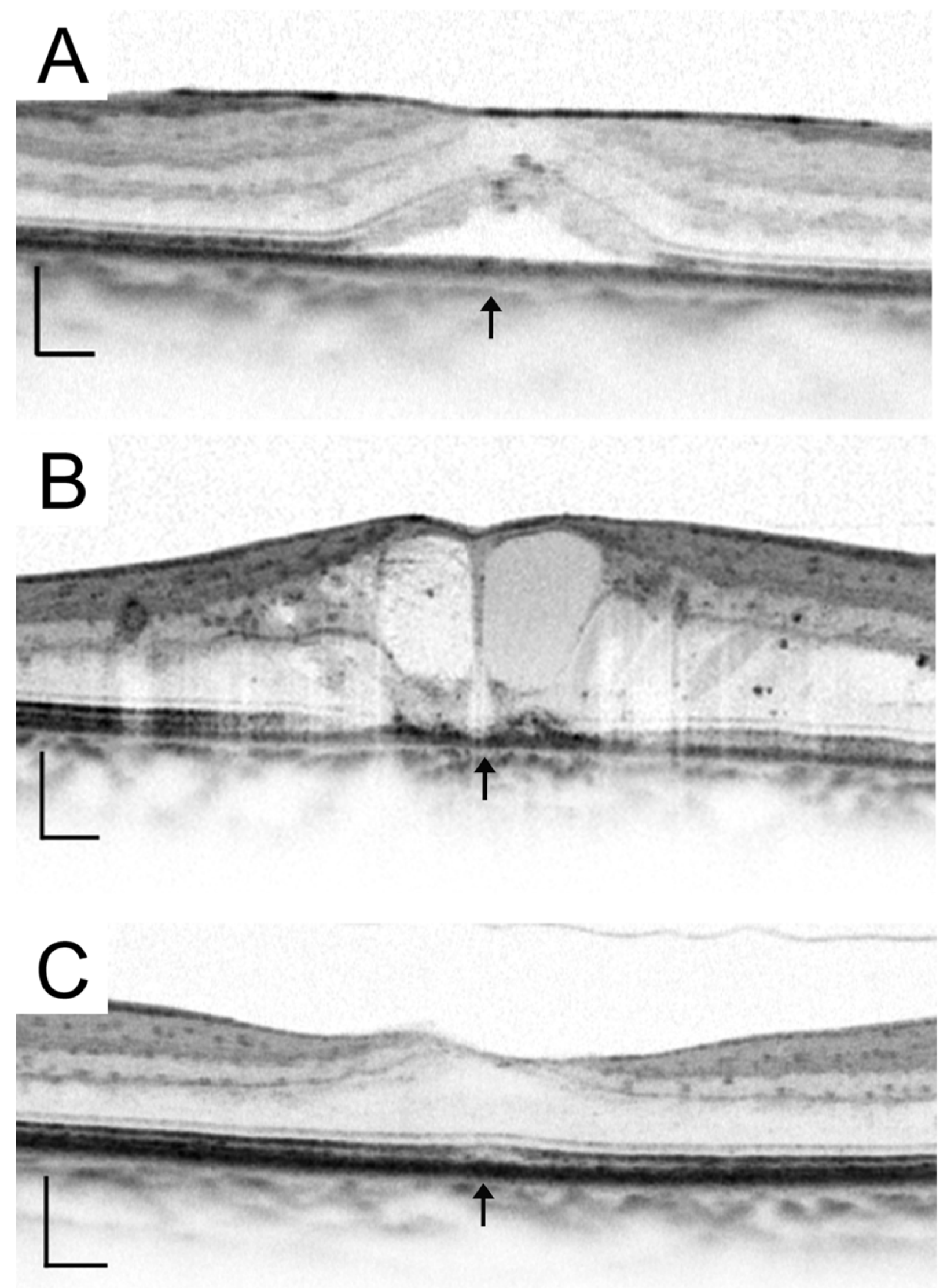


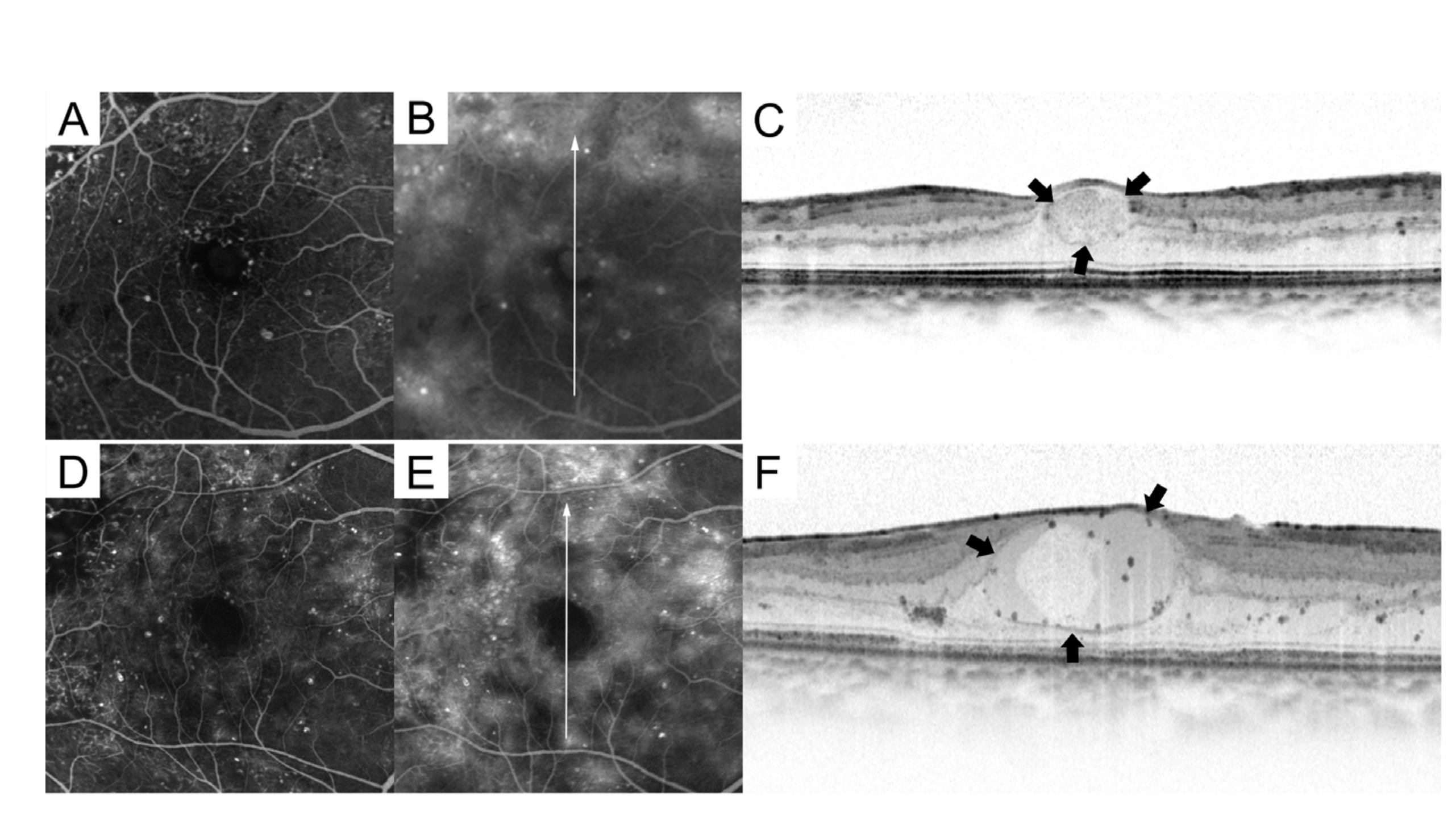

Figure 2.
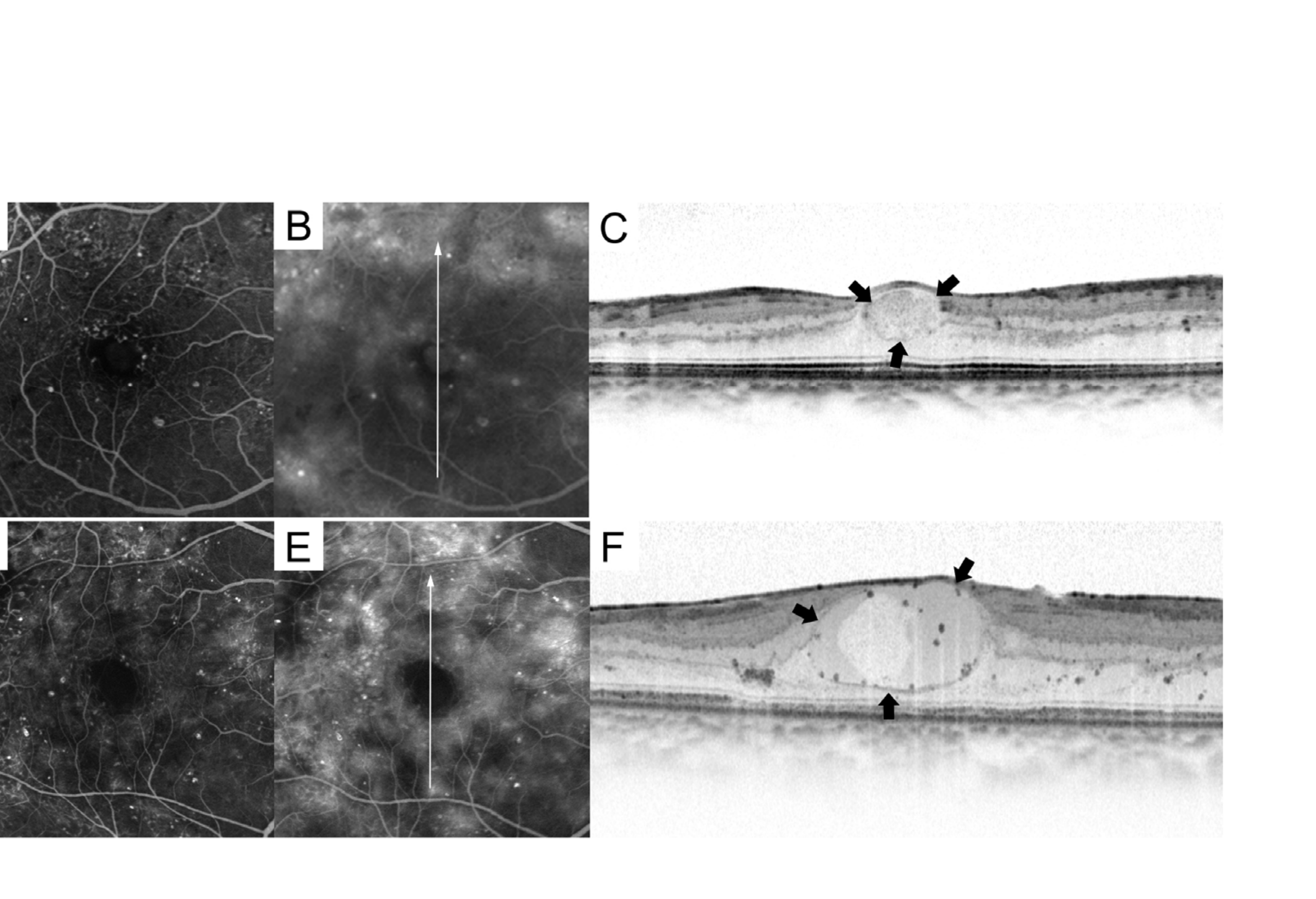

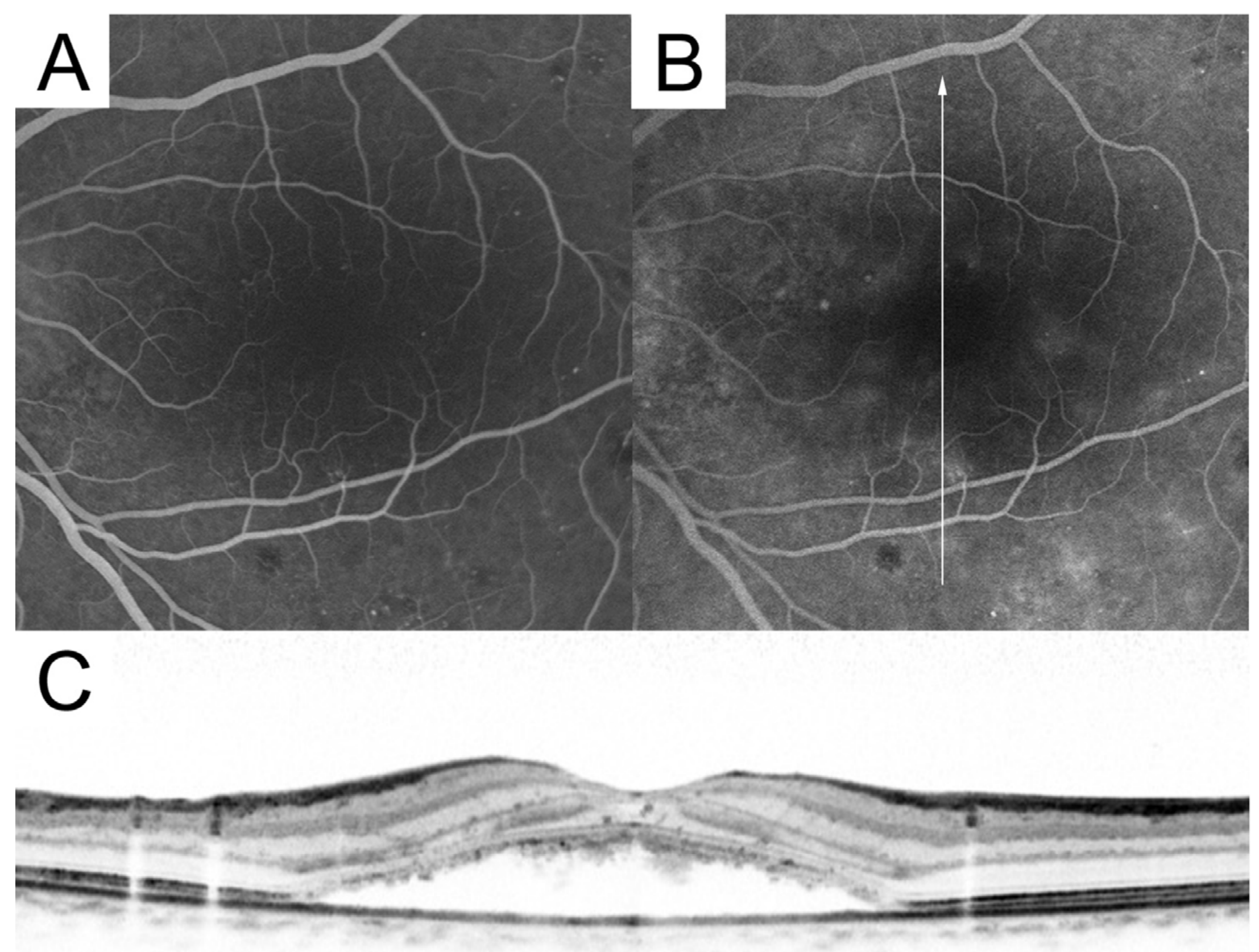


\section{Supplemental Data}

Table 2. Automatically calculated reflectivity levels in 139 cystoid spaces on optical coherence tomography images in diabetic macular edema

\begin{tabular}{c|c|c} 
& $\begin{array}{c}\text { reflectivity levels in cystoid spaces } \\
\text { on OCT images }\end{array}$ & $p$-value \\
\hline $\begin{array}{c}\text { fluorescein pooling } \\
\text { modest }\end{array}$ & $12.6 \pm 9.9$ & $<0.001$ \\
hyperreflecitve foci & $22.7 \pm 16.1$ & $<0.001$ \\
present & & \\
absent & $24.3 \pm 12.6$ & 0.001 \\
microaneurysms in fluorescein pooling & $14.2 \pm 15.1$ & \\
present & $22.8 \pm 11.9$ & \\
absent & $17.0 \pm 15.7$ & 0.464 \\
present & $17.5 \pm 13.7$ &
\end{tabular}

OCT $=$ optical coherence tomography .

$\mathrm{VMT}$ = vitreomacular traction. 